\title{
Criminologie
}

\section{Index cumulatif 1982-1990}

\section{Jacqueline De Plaen}

Volume 24, numéro 2, 1991

Regard sur les tribunaux

URI : https://id.erudit.org/iderudit/017311ar

DOI : https://doi.org/10.7202/017311ar

Aller au sommaire du numéro

Éditeur(s)

Les Presses de l'Université de Montréal

ISSN

0316-0041 (imprimé)

1492-1367 (numérique)

Découvrir la revue

Citer ce document

De Plaen, J. (1991). Index cumulatif 1982-1990. Criminologie, 24(2), 99-124.

https://doi.org/10.7202/017311ar d'utilisation que vous pouvez consulter en ligne.

https://apropos.erudit.org/fr/usagers/politique-dutilisation/ 
INDEX CUMULATIF

\section{CRIMINOLOGIE}

Préparé par:

Jacqueline De Plaen

Agente de recherche, CICC 


\section{INDEX CUMULATIF DES AUTEURS ET COLLABORATEURS 1982-1990}

ACOSTA, Fernando

- Table ronde sur la peine de mort

- À propos des illégalismes privilégiés. Reflexions conceptuelles et mise en contexte

$\begin{array}{rlr}\text { Année } & \text { Vol./n } & \text { Pages } \\ 1987 & \text { XX 2 } & 103-113 \\ 1988 & \text { XXXI 1 } & 7-34 \\ 1988 & \text { XXI 1 } & 95-102 \\ 1982 & \text { XV 2 } & 41-48 \\ & & \\ 1983 & \text { XVI 1 } & 31-49 \\ 1983 & \text { XVI 2 } & 89-100 \\ 1985 & \text { XVII 2 } & 117-133 \\ 1988 & \text { XXI 2 } & 103-111 \\ & & 67-83 \\ 1989 & \text { XXII 1 } & \\ & & 121-125\end{array}$

- L'expertise médicale en matière de faute professionnelle

AUBUT, Jocelyn

- L'évaluation des délinquants sexuels

BARIL, Micheline

- Une illustration de la peur concrète: le cas des victimes

- Quand les femmes sont victimes... quand les hommes appliquent la loi

- Du côté des victimes, une autre perspective sur le vol à main armée

- Les projets de lois et de politiques en matière de victimes d'actes criminels

BEAUCHESNE, Line

- De la criminalisation à la légalisation des drogues: de Charybde en Scylla?

BEAUDRY, Raymonde

- Survol bibliographique de la littérature criminologique féminine

BEAULIEU, Marie

- La conduite en état d'ébriété: les impacts des modifications législatives de décembre 1985 tels que perçus par les policiers

1989 XXII 1

$27-40$

BELLOT, Sylvie

- Les auteurs de vols à main armée à Montréal: une typologie empirique

1985 XVIII 2

BERTRAND, Marie-Andrée

- Femmes et justice: problèmes d'intervention

- Perspectives traditionnelles et perspectives critiques en criminologie

- Résurgence du mouvement antiprohibitionniste

$\begin{array}{rlr}1983 & \text { XVI 2 } & 77-88 \\ 1986 & \text { XIX 1 } & 97-111 \\ 1989 & \text { XXII 1 } & 120-133\end{array}$

BOGOPOLSKY, Yann

- Le clinicien et la question de la «dangerosité» à la croisée du social et de l'individuel 
BRILLON, Yves

- La peur du crime et la punitivité chez les personnes âgées

- L'opinion publique et les politiques criminelles

1986 XIX 1

$227-238$

BRISSETTE, Marc

- La criminalité des affaires et les sentences applicables aux personnes morales

1982

$\mathrm{XV} 1$

$77-104$

BRODEUR, Jean-Paul

- La police: mythes et réalités

- Provocations

1984 XVII $1 \quad 9-41$

1986 XIX $1 \quad 141-169$

COLLETTE-CARRIÈRE, Renée

- Du côté des filles et des femmes, leur délinquance, leur criminalité

- Réflexion autour de la notion de service aux femmes dans le système de justice

XVI 2

1983 XVI 2 101-111

CORDEAU, Gilbert

- Les homicides entre délinquants: une analyse des conflits qui provoquent des règlements de comptes

1989

XXII 2

13-34

- Carrières criminelles collectives : évolution d'une population délinquante (les groupes de motards)

1989 XXII 2

$65-94$

COUSINEAU, Marie-Marthe

- Quand les femmes sont victimes... quand les hommes appliquent la loi

1983 XVI 2

$89-100$

CRELINSTEN, Ronald D.

- La couverture de presse et ses fonctions légitimantes

1987

XX 1

$35-57$

CUSSON, Maurice

- Guide de lecture sur le vol à main armée... si le cœur vous en dit

1985 XVIII 2

- L'analyse stratégique et quelques développements récents en criminologie

- Équivalences pénales et solutions de rechange à l'emprisonnement: la métrique pénale implicite des tribunaux criminels

- Les zones urbaines criminelles

1986 XIX 1

CYR, Caroline

- L'affaire Belmoral

$1988 \quad$ XXI 1

83-94

CYR, Mireille

- Étude de relance auprès des détenus fédéraux traités en milieu psychiatrique: description des antécédents, du séjour, des rechutes et des récidives

DePLAEN, Jacqueline

- Index cumulatif 1968-1981, vol. I-XIV

- Psychiatrie légale et criminalité

\section{DEBUYST, Christian}

- La notion de dangerosité, maladie infantile de la criminologie 
DESMARAIS-GERVAIS, Lise

- La prédiction des contacts avec le tribunal de la jeunesse: utilisation de l'évaluation du comportement par les pairs au début de l'école primaire

1986 XIX 2

$33-48$

DIONNE, Michèle

- La relance des bacheliers en criminologie de 1970 à 1983

$\begin{array}{rrr}1986 & \text { XIX 1 } & 261-279 \\ 1982 & \text { XV 2 } & 7-25 \\ 1984 & \text { XVII 2 } & 25-51 \\ 1983 & \text { XVI 1 } & 85-99 \\ 1985 & \text { XVIII 2 } & 47-67 \\ & & \\ 1990 & \text { XXIII 2 } & 5-22 \\ 1988 & \text { XXI 1 } & 103-119\end{array}$

DOZOIS, Jean

- L'expertise de la dangerosité

- Dangerosité et pratique criminologique en milieu adulte

DURAND, Sylvie

- La peur du crime dans un quartier de Montréal : Côte-des-Neiges

ÉLIE, Daniel

- L'impact de la réaction policière sur

l'évolution de vols avec violence

ENGEL, Frema

- Victimes d'actes criminels: une intervention professionnelle

ÉQUIPE DE CITÉ DES PRAIRIES

- La Cité des Prairies: 25 ans de réalisations

FARRINGTON, David P.

- Les signaux précoces de l'agir délinquant frequent

1986 XIX 2

FERGUSON, Gerry

- Le droit aux soins de santé mentale en milieu carcéral

$1988 \quad$ XXI 2

FORTIN, Jacques

- La commission de réforme du droit du Canada: un bilan succinct

1982 XV 1

$105-112$

FRIGON, Sylvie

- Femmes et héroïne: bilan des connaissances limites et perspectives nouvelles

1989 XXII 1

85-109

GABOR, Thomas

- La couverture du crime par la presse: un portrait fidèle ou déformé?

$1987 \quad X X 1$

$79-98$

GAGNE, Louise

- Où en est la situation de la violence envers les femmes dans le milieu familial, dix ans après les colloques sur la violence?

1990 XXIII 2

GAGNON, Rosette

- Les voleurs à main armée adultes devant les tribunaux, l'étude du cheminement des plaintes 


\section{GALLANT, Gérald}

- Le rapport présentenciel: outil de la prise de décision judiciaire en matière criminelle et pénale

GARCEAU, Marie-Luce

- La détention provisoire au Québec: une pratique juridique courante

GAUTHIER, Maurice

- Les politiques et les pratiques en matière correctionnelle adulte au Québec: 1960-1985

GERMAIN, Daniel

- La femme «psychiatrisée» en milieu carcéral

$1988 \quad$ XXI 2

GERVAIS, Richard

- Soignés, soignants en perte d'équilibre

1988

XXI 2

$91-95$

GRANDBOIS, Maryse

- Le droit pénal de l'environnement: une garantie d'impunité?

1988

XXI 1

$57-81$

GRAVEL, Sylvie

- Quand les femmes sont victimes... quand les hommes appliquent la loi

- Équivalences pénales et solutions de rechange à l'emprisonnement: la métrique pénale implicite des tribunaux criminels

GRAVIER, Bruno

- Approche institutionnelle et impact de la sortie du psychotique dangereux

GRENIER, Bernard

- Le juge et la justice

1982 XV I

HODGINS, Sheilagh

- Institut Philippe-Pinel et la recherche évaluative

- La loi, les procédures et l'histoire des cas

- Étude de relance auprès des détenus fédéraux traités en milieu psychiatrique: description des antécédents, du séjour, des rechutes et des récidives

HOUCHON, Guy

- Évolution du concept de dangerosité en criminologie européenne ( Vingt ans après....»)

KAPÉTANAKI-BARAKÈ, Anne

- L'impact de la réaction policière sur l'évolution de vols avec violence

LABERGE, Danielle

- L'invention de l'enfance: modalités institutionnelles et support idéologique 


\section{LACHANCE, André}

- Le contrôle social dans la société canadienne du Régime français au $X_{V}{ }^{e}{ }^{e}$ siècle

LAFLAMME-CUSSON, Suzanne

- La déclaration de la victime au tribunal: évaluation de l'expérience du palais de justice de Montréal

$\begin{array}{llr}1990 & \text { XXIII 2 } & 73-88 \\ 1990 & \text { XXIII 1 } & 95-116 \\ 1982 & \text { XV 2 } & 105-114 \\ & & \\ 1989 & \text { XXII 2 } & 65-94 \\ 1990 & \text { XXIII 1 } & 3-5 \\ 1990 & \text { XXIII 2 } & 107-123 \\ 1982 & \text { XV 2 } & 7-25 \\ 1984 & \text { XVII 2 } & 25-51\end{array}$

LAFOREST, Suzanne

- Adolescents et délinquance sexuelle

LAGIER, Pierre

- Dangerosité et justice: la peur du criminel ou la peur de la criminologie

LAISNE, Sylvie

- Carrières criminelles collectives: évolution d'une population délinquante (les groupes de motards)

LAJOIE, Jean

- Adolescence et désespoir du sens

- Débat autour des mesures pour jeunes délinquants

LALONDE, Michèle

- L'expertise de la dangerosité

- Dangerosité et pratique criminologique en milieu adulte

LAMOTHE, Pierre

- Problèmes quotidiens de psychiatrie pénitentiaire à travers les changements du paysage psychiatrique et pénitentiaire français

$1988 \quad$ XXI 2

63-81

LAMY, Pierre

- Étude de relance auprès des détenus fédéraux traités en milieu psychiatrique : description des antécédents, du séjour, des rechutes et des récidives

1988 XXI 2

$27-61$

LANDREVILLE, Pierre

- Évolution theorique en criminologie : l'histoire d'un cheminement

- L'alcool au volant, c'est criminel depuis 1921 !

1986 XIX $1 \quad 11-31$

1989 XXII $1 \quad 9-26$

LANGELIER-BIRON, Louise

- Agression et délinquance chez l'adolescente : conduites analogues ou différentes?

- Du côté des filles et des femmes, leur délinquance, leur criminalité

1983 XVI 2

1983 XVI $2 \quad 27-45$

LAPLANTE, Laurent

- L'aide juridique, dix ans après

1982 XV 1

LARRIVÉE, Gaétan

- Le rôle de l'agent de probation avec une clientèle à incidence psychiatrique

LAURENDEAU, Marc

- La police et ses auxiliaires particuliers: informateurs, délateurs et agents provocateurs 
LAROUCHE, Ginette

- Où en est la situation de la violence envers les femmes dans le milieu familial, dix ans après les colloques sur la violence?

LAVERGNE, Chantal

- L'alcool au volant, c'est criminel depuis 1921!

LeBLANC, Marc

- Les policiers, la Loi sur les jeunes contrevenants et le système de justice pour les mineurs

- Le vol à main armée chez les mineurs

- Les voleurs à main armée adultes devant les tribunaux, l'étude du cheminement des plaintes

- La carrière criminelle: définition et prédiction

- Pour une approche intégrative de la conduite délinquante des adolescents

- La prédiction des contacts avec le tribunal de la jeunesse: utilisation de l'évaluation du comportement par les pairs au début de l'école primaire

- Le cycle de la violence physique: trajectoire sociale et cheminement personnel de la violence individuelle et de groupe

- Débat autour des mesures pour jeunes délinquants

LECLERC, Jean-François

- Justice et infrajustice en Nouvelle-France. Les voies de fait à Montréal entre 1700 et 1760

- La Sûreté du Québec des origines à nos jours: quelques repères historiques

1990

XXIII 2

23-45

$1989 \quad$ XXII 1

1984

1985

XVII I

XVIII 2

91-115

7-34

1985

XVIII 2

63-83

1986

XIX 2

79-99

1986 XIX 1

73-95

1986

XIX 2

33-48

1990

XXIII 1

41-74

1990

XXIII 2

$107-123$

1985

XVIII 1

25-42

1989 XXII $2 \quad 107-127$

LECOR, Josée

- La peur du crime, dépossession de soi

1983

XVI 1

$101-103$

LEFEBVRE, Yvon

- La loi, les procédures et l'histoire des cas

1982

XV 2

49-81

LÉGARÉ, Jocelyne

- La condition juridique des femmes ou l'historique d'une "affaire de famille»

1983 XVI 2

LEGUERRIER, Yves

- Les entreprises de prostitution commerciale: les commerces éphémères des marchés illicites

1989

XXII 2

$35-63$

LEMIRE, Guy

- Théories et pratiques criminologiques: quand l'organisation mène le jeu

198

XIX 1

$215-226$

LEROUX, Josette

- Le rôle de l'agent de probation avec une clientèle à incidence psychiatrique

XXI 2

LEVY, René

- Police, État, insécurité
1984 XVII 1 
LIPPEL, Katherine

- Les victimes sans crimes: le traitement pénal des accidents du travail

\begin{tabular}{|c|c|c|}
\hline 1988 & XXI 1 & $35-56$ \\
\hline 1986 & XIX 2 & $49-77$ \\
\hline 1983 & XVI 2 & $47-66$ \\
\hline 1987 & XX 1 & $7-33$ \\
\hline 1983 & XVI 1 & $69-83$ \\
\hline 1983 & XVI 1 & $105-106$ \\
\hline 1989 & XXII 2 & 65-94 \\
\hline 1986 & XIX 2 & $101-115$ \\
\hline 1989 & XXII 1 & $41-65$ \\
\hline 19 & XXIII 1 & $7-39$ \\
\hline
\end{tabular}

OEBER, Rolf

- La prédiction de la délinquance

LOS, Maria

- Les femmes, le pouvoir et le crime en Pologne

- La Loi sur les jeunes contrevenants et les mass-médias

LOUIS-GUÉRIN, Christiane

- La peur du crime: mythes et réalités

MacKAY, Pierre

- Ce soir au Grand Guignol : l'étrangleur de Boston

MacLEAN, Brian

- Carrières criminelles collectives: évolution d'une population délinquante (les groupes de motards)

MARCEAU, Bruno

- La prédiction du comportement violent: exercice nécessaire et délicat

MITCHELLL, Chet M.

- Le crime organisé et la guerre aux stupéfiants: crise et reforme

MESSIER, Camille

- Les troubles de comportement à l'adolescence et leur traitement en centre d'accueil à la suite d'une ordonnance de protection

MORRISSETTE, Anne

- Du côté des victimes, une autre perspective sur le vol à main armée

1985 XVIII $2 \quad 117-133$

MURBACH, Ruth

- Note de recherche: enjeux de la peine et efficacité sociojuridique, le cas du braconnage

$1987 \quad X X 2$

89-102

NORMANDEAU, André

- Dangerosité et justice: la peur du criminel ou la peur de la criminologie

- Politiques pénales et peur du crime

- Guide de lecture sur le vol à main armée... si le coeur vous en dit

- Stratégies de changement et politiques pénales

PAQUET, Jean

- Étude de relance auprès des détenus fédéraux traités en milieu psychiatrique: description des antécédents, du séjour, des rechutes et des récidives

PARADIS, Richard

- Adolescents et délinquance sexuelle 
PARENT, Georges-André

- Presse et corps policiers: complicité et conflit

1987

$\mathrm{XX} 1$

$99-120$

- Les médias: source de victimisation

1990

XXIII 2

PARIZEAU, Alice

- Le rapport présentenciel et les politiques criminelles au Québec

- L'individu et la collectivité

1982

$X V_{1}$

1982

XV 2

115-122

- Les problèmes éthiques, sociaux et légaux de la psychiatrie

1982 XV 2

PINSONNEAULT, Pierre

- L'abandon de la carrière criminelle : quelques témoignages

XVIII $2 \quad 85-116$

PIRES, Alvaro P.

- La réforme pénale au Canada: l'apport de la Commission canadienne sur la détermination de la peine

POIRIER, Donate

- Prévenir le vol à main armée?

198

XVIII 2 135-145

POIRIER, Robert

- La négociation des sentences du point de vue des avocats de la défense

$57-68$

POUPART, Jean

- L'expertise de la dangerosité

1982 XV 2

- Dangerosité et pratique criminologique en milieu adulte

1984 XVII 2

QUINZEY, Vernon L.

- Politique institutionnelle de liberation: identification des individus dangereux. Une revue de littérature

1984 XVIl 2

53-78

RAINS, Prue

- La justice des mineurs et The Boys's Farm: 1909-1968

RICO, José M.

- Le droit de punir

1986 XIX 1

$113-140$

ROBERT, Philippe

- Police, État, insécurité

1984 XVII 1

43-58

ROBERT, Pierre

- Le procès criminel: éléments d'une approche sociojuridique de la procédure pénale

$1982 \quad \mathrm{XV} 1$

RONDEAU, Roger

- Violents jusqu'au meurtre? Réflexions sur deux cas d'homicide

$1990 \quad$ XXIII 1

SCHWARTZMAN, Alexandre

- La prediction des contacts avec le tribunal de la jeunesse: utilisation de l'évaluation du comportement par les pairs au début de l'école primaire 
SHEARING, Clifford D.

- La sécurité privée au Canada: quelques questions et réponses

1984

XVII I

SHEWSHUCK, Angela

- Carrières criminelles collectives: évolution d'une population délinquante (les groupes de motards)

1989

XXII 2

SOUBIRAN-PAILLET, Francine

- Presse et délinquance ou Comment lire entre les signes

$\mathrm{XX} 1$

STOUTHAMER-LOEBER, Magda

- La prédiction de la délinquance

1986 XIX 2

SURPRENANT, André

- Approche institutionnelle et impact de la sortie du psychotique dangereux

1982 XV 2

SZABO, Denis

- Entre l'arbre et l'écorce: la recherche criminologique au Canada (1960-1985)

THERRIAULT, Guy

- La punition commune du crime: la prison et l'amende à Montréal de 1845 à 1913

1985 XVIII 1

TREMBLAY, Pierre

- La punition commune du crime: la prison et l'amende à Montréal de 1845 à 1913

- Équivalences pénales et solutions de rechange à l'emprisonnement: la métrique pénale implicite des tribunaux criminels

- Plaidoyer pour la sociologie criminelle

- Carrières criminelles collectives: évolution d'une population délinquante (les groupes de motards)

1986 XIX 1

$1985 \quad$ XVIII 1

$1987 \quad X X 2$

1989 XXII 2

69-88

$1989 \quad$ XXII $2 \quad 65-94$

TREMBLAY, Richard E.

- La prédiction des contacts avec le tribunal de la jeunesse: utilisation de l'évaluation du comportement par les pairs au début de l'école primaire

1986 XIX 2

TRÉPANIER, Jean

- La justice des mineurs au Québec: 25 ans de transformations (1960-1985)

$1986 \quad$ XIX $1 \quad 189-213$

TRUDEAU-LeBLANC, Pierrette

- Hommage à Denis Szabo

1986 XIX 1

TURCOTTE, Lise

- La communauté thérapeutique du pénitencier Donnacona: un an déjà!

$1989 \quad$ XXII $1 \quad 111-119$

VALCOURT, Dianne

- La Maison Thérèse-Casgrain, une ressource communautaire

1983 XVI $2 \quad 113-119$

VANASSE, Cécile

- Agression et délinquance chez l'adolescente: conduites analogues ou différentes? 
VIGNOLA, Henri-Paul

- La police, un service municipal comme les autres

1984 XVII I 127-131

WALLER, Irvin

- L'enjeu des années 1990: la police, les tribunaux et les municipalités

1990 XXIII 2 89-105

WEIMANN, Gabriel

- La couverture du crime par la presse: un portrait fidèle ou déformé?

$1987 \quad X X 1$

79-98 


\section{INDEX DES SUJETS \\ 1982-1990}

Année Vol./no Pages

BIBLIOGRAPHIES ET NOTES DE LECTURE

- Survol bibliographique de la littérature criminologique féminine Raymonde BEAUDRY

- Index cumulatif 1968-1981, vol. I-XIV Jacqueline DePLAEN

- Psychiatrie légale et criminalité Jacqueline DePLAEN

- Dangerosité et justice : la peur du criminel ou la peur de la criminologie Pierre LAGIER et André NORMANDEAU

- Guide de lecture sur le vol à main armée... si le coeur vous en dit André NORMANDEAU et Maurice CUSSON

- L'individu et la collectivité Alice PARIZEAU

1983 XVI $2 \quad 121-125$

$1982 \quad$ XV I $\quad 123-146$

1982 XV $2 \quad 131-134$

1982 XV $2 \quad 105-114$

$1985 \quad$ XVIII $2 \quad 147-154$

1982 XV $2 \quad 115-122$

1982 XV $2 \quad 123-129$ la psychiatrie Alice PARIZEAU

\section{CARRIËRE CRIMINELLE}

- La carrière criminelle: définition et prédiction Marc LeBLANC

1986 XIX 2

$79-99$

- L'abandon de la carrière criminelle: quelques tếmoignages Pierre PINSONNEAULT

1985 XVIII $2 \quad 85-116$

- Carrières criminelles collectives : évolution d'une population délinquante (les groupes de motards)

Pierre TREMBLAY,

Sylvie LAISNE,

Gilbert CORDEAU,

Brian MacLEAN et

Angela SHEWSHUCK

\section{CRIME ÉCONOMIQUE}

- La criminalité des affaires et les sentences applicables aux personnes morales Marc BRISSETTE
1982 XV $1 \quad 77-104$ 
- L'affaire Belmoral

1988

XXI 1

83-94

Caroline CYR

- Le droit pénal de l'environnement : une garantie d'impunité?

1988

XXI 1

$57-81$ Maryse GRANDBOIS

- Les entreprises de prostitution commerciale: les commerces éphémères des marchés illicites Yves LEGUERRIER

$1989 \quad$ XXII 2

$35-63$

- Les victimes sans crimes: le traitement pénal des accidents du travail

$1988 \quad$ XXI 1

$35-56$

Katherine LIPPEL

\section{CRIME ORGANISÉ}

- Les homicides entre délinquants : une analyse des conflits qui provoquent des règlements de comptes

Gilbert CORDEAU

- Le crime organisé et la guerre aux stupéfiants : crise et réforme Chet M. MITCHELL

- Carrières criminelles collectives: évolution d'une population délinquante (les groupes de motards)

PierTe TREMBLAY,

Sylvie LAISNE,

Gilbert CORDEAU,

Brian MacLEAN et

Angela SHEWSHUCK

\section{CRIMINOLOGIE: THÉORIE, RECHERCHE}

\section{ET CRITIQUE}

- À propos des illégalismes privilégiés. Réflexions conceptuelles et mise en contexte Fernando ACOSTA

- Perspectives traditionnelles et perspectives critiques en criminologie Marie-Andrée BERTRAND

- Provocations

1986 XIX $1 \quad 97-111$ Jean-Paul BRODEUR

1986 XIX $1 \quad 141-169$

- L'analyse stratégique et quelques développements récents en criminologie Maurice CUSSON

1986 XIX $1 \quad 53-72$

- Évolution théorique en criminologie : l'histoire d'un cheminement

1986 XIX 1 Pierre LANDREVILLE

- Pour une approche intégrative de la conduite délinquante des adolescents 


\section{CRIMINOLOGUES}

- La relance des bacheliers en criminologie de 1970 à 1983 Michèle DIONNE

- Hommage à Denis Szabo Pierrette TRUDEAU-LeBLANC

1986 XIX 1

\section{DANGEROSITÉ}

- Le clinicien et la question de la "dangerosité» à la croisée du social et de l'individue] Yann BOGOPOLSKY

1984 XVII $2 \quad 93-101$

- La notion de dangerosité, maladie infantile de la criminologie Christian DEBUYST

1984 XVII $2 \quad 7-24$

- Dangerosité et pratique criminologique en milieu adulte Jean DOZOIS, Michèle LALONDE et Jean POUPART

- Évolution du concept de dangerosité en criminologie européenne ( Vingt ans après...") Guy HOUCHON

1984 XVII $2 \quad 79-91$

- Dangerosité et justice: la peur du criminel ou la peur de la criminologie Pierre LAGIER et André NORMANDEAU

- L'expertise de la dangerosité Jean POUPART, Jean DOZOIS et Michèle LALONDE

- Politique institutionnelle de libération: identification des individus dangereux. Une revue de littérature Vemon L. QUINZEY

1984 XVII $2 \quad 25-51$

1982 XV $2 \quad 105-114$

1982 XV $2 \quad 7.25$

1984 XVII $2 \quad 53-78$

\section{FEMMES: DÉLINQUANCE ET JUSTICE}

- Quand les fermmes sont victimes... quand les hommes appliquent la loi

1983 XVI $2 \quad 89-100$ Micheline BARIL, Marie-Marthe COUSINEAU et Sylvie GRAVEL

- Survol bibliographique de la littérature criminologique féminine Raymonde BEAUDRY

- Femmes et justice: problèmes d'intervention Marie-Andrée BERTRAND

- Réflexion autour de la notion de service aux femmes dans le système de justice Renée COLLETTE-CARRIÈRE

1983 XVI $2 \quad 121-125$

1983 XVI $2 \quad 77-88$

1983 XVI $2 \quad 101-111$ 
- Du côté des filles et des femmes, leur délinquance, leur criminalité Renée COLLETTE-CARRIËRE et Louise LANGELIER-BIRON

- Femmes et hérojne: bilan des connaissances limites et perspectives nouvelles Sylvie FRIGON

- La femme "psychiatrisée» en milieu carcéral Daniel GERMAIN

1983

XVI 2

$27-45$

Agression et délinquance chez l'adolescente: conduites analogues ou différentes?

Louise LANGELIER-BIRON et Cécile VANASSE

- Où en est la situation de la violence envers les femmes dans le milieu familial, dix ans après les colloques sur la violence?

Ginette LAROUCHE et Louise GAGNÉ

- La condition juridique des femmes ou I'historique d'une «affaire de famille" Jocelyne LEGARÉ

- Les femmes, le pouvoir et le crime en Pologne Maria LOS

- La Maison Thérèse-Casgrain, une ressource communautaire Dianne VALCOURT

1989 XXII

$1988 \quad$ XXI 2

$97-102$

1983 XVI 2

$67-76$

1990

XXIII 2

23.45

1983 XVI 2

1983 XVI 2

$47-66$

1983 XVI 2

113-119

\section{DÉLINQUANTS MINEURS}

- Les signaux précoces de l'agir délinquant fréquent

1986

XIX 2

David P. FARRINGTON

- Adolescents et délinquance sexuelle Suzanne LAFOREST et Richard PARADIS

- Adolescence et désespoir du sens Jean LAJOIE

1990

XXIII

$95-116$

$1990 \quad$ XXIII 1

- Agression et délinquance chez l'adolescente: conduites analogues ou différentes?

1983 XVI 2

Louise LANGELIER-BIRON et Cécile VANASSE

- Le vol à main armée chez les mineurs Marc LeBLANC

1985 XVIII 2

- Pour une approche intégrative de la conduite délinquante des adolescents

1986 XIX 1 Marc LeBLANC

- Le cycle de la violence physique: trajectoire sociale et cheminement personnel de la violence individuelle et de groupe Marc LeBLANC 
- La prédiction de la délinquance

1986

XIX 2

$49-77$

Rolf LOEBER et

Magda STOUTHAMER-LOEBER

- Violents jusqu'au meurtre? Réflexions sur deux cas d'homicide

Roger RONDEAU

$1990 \quad$ XXIII I

$75-93$

\section{DETERMINATION DE LA SENTENCE}

- Le juge et la justice

1982 XV I

113-116 Bernard GRENIER

- Note de recherche: enjeux de la peine et efficacité sociojuridique, le cas du braconnage Ruth MURBACH

$1987 \quad X X 2 \quad 89-102$

- La réforme pénale au Canada: l'apport de la Commission canadienne sur la détermination de la peine

Alvaro P. PIRES

- La négociation des sentences du point de vue des avocats de la défense Robert POIRIER

- Équivalences pénales et solutions de rechange à l'emprisonnement: la métrique pénale implicite des tribunaux criminels

Pierre TREMBLAY,

Sylvie GRAVEL et

Maurice CUSSON

\section{DROGUE ET ALCOOL}

- De la criminalisation à la légalisation des drogues: de Charybde en Scylla?

Line BEAUCHESNE

$1989 \quad$ XXII 1

$67-83$

- La conduite en état d'ébriété: les impacts des modifications législatives de décembre 1985 tels que perçus par les policiers Marie BEAULIEU

- Résurgence du mouvement antiprohibitionniste Marie-Andrée BERTRAND

$1987 \quad X X 2$

$1987 \quad$ XX 2

$57-68$

$1987 \quad \times X 2 \quad 69-88$

- Femmes et héroïne : bilan des connaissances limites et perspectives nouvelles Sylvie FRIGON

- L'alcool au volant, c'est criminel depuis 1921! Pierre LANDREVILLE et Chantal LAVERGNE

- Le crime organisé et la guerre aux stupéfiants: crise et réforme Chet M. MITCHELL

1989 XXII $1 \quad 27-40$

$1989 \quad$ XXII $1 \quad 120-133$

1989 XXII $1 \quad 85-109$

1989 XXII $1 \quad 9-26$

- Le droit de punir

1986 XIX 1 113-140

José M. RICO 


\section{DROIT PÉNAL}

- Femmes et justice: problèmes d'intervention

1983

XVI 2

$77-88$ Marie-Andrée BERTRAND

- La commission de reforme du droit du Canada : un bilan succinct Jacques FORTIN

1982 XV

$105-112$

- Le droit pénal de l'environnement: une garantie d'impunité? Maryse GRANDBOIS

- Le droit de punir José M. RICO

\section{ÉTUDE HISTORIQUE}

- L'invention de l'enfance: modalités institutionnelles et support idéologique Danielle LABERGE

1985 XVIII 1

73-97

- Le contrôle social dans la société canadienne du Régime français au XVIII ${ }^{e}$ siècle

1985 XVIII 1 André LACHANCE

- Justice et infrajustice en Nouvelle-France. Les voies de fait à Montréal entre 1700 et 1760 Jean-François LECLERC

1985 XVIII 1

- La Sûreté du Québec des origines à nos jours : quelques repères historiques Jean-François LECLERC

- La condition juridique des femmes ou l'historique d'une «affaire de famille» Jocelyne LEGARÉ

- La justice des mineurs et The Boys's Farm: 1909-1968 Prue RAINS

- La punition commune du crime: la prison et l'amende à Montréal de 1845 à 1913

Pierre TREMBLAY et Guy THERRIAULT

\section{ÉVALUATION ET TRAITEMENT}

- L'évaluation des délinquants sexuels Jocelyn AUBUT

- La femme «psychiatrisée» en milieu carcéral Daniel GERMAIN

- Approche institutionnelle et impact de la sortie du psychotique dangereux

- Institut Philippe-Pinel et la recherche évaluative Sheilagh HODGINS 
- Étude de relance auprès des détenus fédéraux traités en milieu psychiatrique: description des antécédents, du séjour, des rechutes et des récidives

Sheilagh HODGINS,

Mireille CYR,

Jean PAQUET et

Pierre LAMY

- La loi, les procédures et l'histoire des cas Sheilagh HODGINS, Yvon LEFEBVRE et coll.

- Problèmes quotidiens de psychiatrie pénitentiaire à travers les changements du paysage psychiatrique et pénitentiaire français Pierre LAMOTHE

1988 XXI 2

63-81

- Le rôle de l'agent de probation avec une clientèle à incidence psychiatrique Josette LEROUX et Gaétan LARRIVÉE

- L'expertise de la dangerosité Jean POUPART, Jean DOZOIS et Michèle LALONDE

- La communauté thérapeutique du pénitencier Donnacona: un an déjà! Lise TURCOTTE

FAUTE PROFESSIONNELLE

- L'expertise médicale en matière de faute professionnelle

$1988 \quad$ XXI 1

95-102

Fernando ACOSTA

\section{HISTOIRE DE CAS}

- Oreste ou l'impossible réparation Pierre LABERGE

- La peur du crime, dépossession de soi Josée LECOR

1989 XXII $1 \quad 111-119$

Le rôle de l'agent de probation avec une clientèle à incidence psychiatrique Josette LEROUX et Gaétan LARRIVÉE

$\begin{array}{rrr}1982 & \text { XV 2 } & 27-40 \\ 1983 & \text { XVI 1 } & 101-103\end{array}$

$1988 \quad$ XXI $2 \quad 83-89$

\section{JUSTICE ET INSTITUTION POUR MINEURS}

- La Cité des Prairies: 25 ans de réalisations ÉQUIPE DE CITÉ DES PRAIRIES

- L'invention de l'enfance: modalités institutionnelles et support idéologique Danielle LABERGE

- Adolescents et délinquance sexuelle Suzanne LAFOREST et Richard PARADIS 
- Débat autour des mesures pour jeunes délinquants.

$1990 \quad$ XXIII 2

$107-123$

Jean LAJOIE et

Marc LeBLANC

- Les policiers, la Loi sur les jeunes

contrevenants et le système de justice pour les mineurs

Marc LeBLANC

1984 XVII 1

$91-115$

- La Loi sur les jeunes contrevenants et les mass-médias

Maria LOS

- Les troubles de comportement à l'adolescence et leur traitement en centre d'accueil à la suite d'une ordonnance de protection Camille MESSIER

- La prédiction des contacts avec le tribunal de la jeunesse: utilisation de l'évaluation du comportement par les pairs au début de l'école primaire

Richard E. TREMBLAY, Lise DESMARAIS-GERVAIS,

Marc LeBLANC et Alexandre SCHWARTZMAN

- La justice des mineurs au Québec: 25 ans de transformations (1960-1985) Jean TRÉPANIER

1986 XIX $2 \quad 33-48$

$1986 \quad$ XIX $1 \quad 189-213$

\section{LOIS, POLITIQUES ET PRATIQUES PÉNALES}

- Les projets de lois et de politiques en matière de victimes d'actes criminels Micheline BARIL

- De la criminalisation à la légalisation des drogues: de Charybde en Scylla?

$1989 \quad$ XXII 1 Line BEAUCHESNE

- La conduite en état d'ébriété: les impacts des modifications législatives de décembre 1985 tels que perçus par les policiers Marie BEAULIEU

- Résurgence du mouvement antiprohibitionniste Marie-Andrée BERTRAND

- L'opinion publique et les politiques criminelles Yves BRILLON

1989 XXII 1

La détention provisoire au Québec: une pratique juridique courante Marie-Luce GARCEAU

1989 XXII 1 120-133

1986 XIX $1 \quad 227-238$

$1990 \quad$ XXIII $1 \quad 117-134$

- Les politiques et les pratiques en matière correctionnelle adulte au Québec: 1960-1985 Maurice GAUTHIER

- La loi, les procédures et l'histoire des cas 
- La déclaration de la victime au tribunal: évaluation de l'expérience du palais de justice de Montréal

1990 XXIII 2

73-88

Suzanne LAFLAMME-CUSSON

- L'alcool au volant, c'est criminel depuis 1921! Pierre LANDREVILLE et

1989 XXII $1 \quad 9-26$ Chantal LAVERGNE

- Theories et pratiques criminologiques: quand l'organisation mène le jeu Guy LEMIRE

- Politiques pénales et peur du crime André NORMANDEAU

- Stratégies de changement et politiques pénales André NORMANDEAU

- Le rapport présentenciel et les politiques criminelles au Québec Alice PARIZEAU

- Entre l'arbre et l'écorce: la recherche criminologique au Canada (1960-1985) Denis SZABO

$\begin{array}{rrr}1986 & \text { XIX 1 } & 215-226 \\ 1983 & \text { XVI 1 } & 51-68 \\ 1986 & \text { XIX 1 } & 171-187 \\ 1982 & \text { XV 1 } & 51-75 \\ 1986 & \text { XIX 1 } & 33-52\end{array}$

\section{MÉDIAS}

- La couverture de presse et ses fonctions légitimantes Ronald D. CRELINSTEN

$1987 \quad X \times 1$

- La couverture du crime par la presse : un portrait fidèle ou déformé?

Thomas GABOR et Gabriel WEIMANN

$\begin{array}{lll}1987 & X X 1 & 79-98\end{array}$

- La Loi sur les jeunes contrevenants et les mass-médias Maria LOS

- Presse et corps policiers : complicité et conflit Georges-André PARENT

- Les médias: source de victimisation Georges-André PARENT

- Presse et délinquance ou Comment lire entre les signes

Francine SOUBIRAN-PAILLET

$\begin{array}{rlr}1987 & \text { XX 1 } & 7-33 \\ 1987 & \text { XX 1 } & 99-120 \\ 1990 & \text { XXIII 2 } & 47-71 \\ & & \\ 1987 & \text { XX 1 } & 59-77\end{array}$

\section{PEINE DE MORT}

- Table ronde sur la peine de mort

Fernando ACOSTA

\section{PERSONNEL JUDICIAIRE ET CORRECTIONNEL}

- Le juge et la justice

1982 XV I

$113-116$ Bernard GRENIER

- Le rôle de l'agent de probation avec une clientèle à incidence psychiatrique 
- La négociation des sentences du point de vue des avocats de la défense

Robert POIRIER

XX 2

$57-68$

\section{PEUR DU CRIME}

- Une illustration de la peur concrète : le cas des victimes Micheline BARIL

$1983 \quad$ XVI $1 \quad 31-49$

- La peur du crime et la punitivité chez les personnes âgées Yves BRILLON

1983 XVI 1

- La peur du crime dans un quartier de Montréal : Côte-des-Neiges Sylvie DURAND

- La peur du crime, dépossession de soi Josée LECOR

- La peur du crime: mythes et réalités Christiane LOUIS-GUÉRIN

- Ce soir au Grand Guignol: l'étrangleur de Boston Pierre MacKAY

- Politiques pénales et peur du crime André NORMANDEAU

$\begin{array}{rrr}1983 & \text { XVI 1 } & 85-99 \\ 1983 & \text { XVI 1 } & 101-103 \\ 1983 & \text { XVI 1 } & 69-83 \\ & & \\ 1983 & \text { XVI 1 } & 105-106 \\ 1983 & \text { XVI 1 } & 51-68\end{array}$

\section{POLICE}

- La conduite en êtat d'ébriété: les impacts des modifications législatives de décembre 1985 tels que perçus par les policiers Marie BEAULIEU

- La police: mythes et réalités Jean-Paul BRODEUR

- L'impact de la réaction policière sur l'évolution de vols avec violence Daniel ÉLIE et Anne KAPÉTANAKI-BARAKÈ

- La police et ses auxiliaires particuliers : informateurs, délateurs et agents provocateurs Marc LAURENDEAU

$1989 \quad$ XXII $1 \quad 27-40$

1984 XVII 1

$1985 \quad$ XVIII $2 \quad 47-67$

- Les policjers, la Loi sur les jeunes contrevenants et le système de justice pour les mineurs

1984 XVII I 91-115 Marc LeBLANC

- La Sûreté du Québec des origines à nos jours: quelques repères historiques Jean-François LECLERC

$1989 \quad$ XX1I $2 \quad 107-127$

- Police, État, insécurité

1984 XVII 1

43-58

René LÉVY et Philippe ROBERT

- Presse et corps policiers: complicité et conflit Georges-André PARENT 
- La sécurité privée au Canada: quelques questions et réponses

1984 XVII I

59-89

Clifford D. SHEARING

- La police, un service municipal comme les autres

Henri-Paul VIGNOLA

1984 XVII I

$127-131$

\section{PRÉDICTION}

- Les signaux précoces de l'agir délinquant fréquent David P. FARRINGTON

- La carrière criminelle: définition et prédiction Marc LeBLANC

- Le cycle de la violence physique: trajectoire sociale et cheminement personnel de la violence individuelle et de groupe Marc LeBLANC

- La prédiction de la délinquance Rolf LOEBER et Magda STOUTHAMER-LOEBER

- La prédiction du comportement violent: exercice nécessaire et délicat Bruno MARCEAU

- L'expertise de la dangerosité Jean POUPART, Jean DOZOIS et Michèle LALONDE

- Politique institutionnelle de libération: identification des individus dangereux. Une revue de littérature Vernon L. QUINZEY

- La prédiction des contacts avec le tribunal de la jeunesse: utilisation de l'évaluation du comportement par les pairs au début de l'école primaire

Richard E. TREMBLAY, Lise DESMARAIS-GERVAIS, Marc LeBLANC et

\section{Alexandre SCHWARTZMAN}

\section{PSYCHIATRIE PÉNITENTIAIRE}

- Psychiatrie légale et criminalité Jacqueline DePLAEN

- Le droit aux soins de santé mentale en milieu carcéral Gerry FERGUSON

- Soignés, soignants en perte d'équilibre Richard GERVAIS

- La loi, les procédures et l'histoire des cas Sheilagh HODGINS, Yvon LEFEBVRE et coll.

1982 XV 2

$131-134$

$1988 \quad$ XXI 2

$1988 \quad$ XXI 2

$91-95$

1982 XV 2

49-81 
- Problèmes quotidiens de psychiatrie pénitentiaire à travers les changements du paysage psychiatrique et pénitentiaire français Pierre LAMOTHE

- L'individu et la collectivité

1982 XV 2 Alice PARIZEAU

- Les problèmes éthiques, sociaux et légaux de la psychiatrie Alice PARIZEAU

1982 XV 2 123-129

\section{RAPPORT PRÉSENTENCIEL}

- Le rapport présentenciel: outil de la prise de décision judiciaire en matière criminelle et pénale Gérald GALLANT

$1982 \quad \mathrm{XV} J \quad 39-49$

- Le rapport présentenciel et les politiques criminelles au Québec Alice PARIZEAU

\section{SOCIOLOGIE DU MILIEU CRIMINEL}

- Les zones urbaines criminelles Maurice CUSSON

- Plaidoyer pour la sociologie criminelle Pierre TREMBLAY

$1989 \quad$ XXII $2 \quad 5-12$

\section{SYSTÈME PÉNAL}

- Réflexion autour de la notjon de service aux femmes dans le système de justice Renée COLLETTE-CARRIÈRE

- L'aide juridique, dix ans après Laurent LAPLANTE

- Le procès criminel : éléments d'une approche sociojuridique de la procédure pénale Pierre ROBERT

\section{VICTIME}

- Une illustration de la peur concrète: le cas des victimes Micheline BARIL

- Du côté des victimes, une autre perspective sur le vol à main armée

1985 XVIII $2 \quad 117-133$ Micheline BARIL

- Les projets de lois et de politiques en matière de victimes d'actes criminels Micheline BARIL

- Quand les femmes sont victimes... quand les hommes appliquent la loi

Micheline BARIL.

Marie-Marthe COUSINEAU et Sylvie GRAVEL 
- La peur du crime et la punitivité chez les personnes âgées Yves BRILLON

$\begin{array}{llr}1983 & \text { XVI 1 } & 7-29 \\ 1988 & \text { XXI 1 } & 83-94 \\ 1990 & \text { XXIII 2 } & 5-22 \\ 1990 & \text { XXIII 2 } & 73-88 \\ & & \\ 1990 & \text { XXIII 2 } & 23-45 \\ & & \\ 1988 & \text { XXI 1 } & 35-56 \\ 1990 & \text { XXIII 2 } & 47-71 \\ & & 89-105 \\ 1990 & \text { XXIII 2 } & \end{array}$

- L'affaire Belmoral Caroline CYR

- Victimes d'actes criminels: une intervention professionnelle Frema ENGEL

- La déclaration de la victime au tribunal: évaluation de l'expérience du palais de justice de Montréal

\section{Suzanne LAFLAMME-CUSSON}

- Où en est la situation de la violence envers les femmes dans le milieu familial, dix ans après les colloques sur la violence?

Ginette LAROUCHE et Louise GAGNÉ

- Les victimes sans crimes: le traitement pénal des accidents du travail Katherine LIPPEL

- Les médias: source de victimisation Georges-André PARENT

- L'enjeu des années 1990: la police, les tribunaux et les municipalités Irvin WALLER

\section{VOL À MAIN ARMÉE}

- Du côté des victimes, une autre perspective sur le vol à main armée

1985 XVIII $2 \quad 117-133$ Micheline BARIL

- Les auteurs de vols à main armée à Montréal: une typologie empirique Sylvie BELLOT

- L'impact de la réaction policière sur l'évolution de vols avec violence Daniel ÉLIE et Anne KAPÉTANAKI-BARAKÈ

- Les voleurs à main arnée adultes devant les tribunaux, l'étude du cheminement des plaintes Rosette GAGNON et Marc LeBLANC

- Le vol à main armée chez les mineurs Marc LeBLANC

- Guide de lecture sur le vol à main armée... si le cœur vous en dit

André NORMANDEAU et Maurice CUSSON

- L'abandon de la carrière criminelle: quelques témoignages Pierre PINSONNEAULT 
- Prévenir le vol à main armée?

1985. XVIIl 2

$135-145$ Donate POIRIER 